\title{
THE MULTIPLY-CHARGED PRIMARY COSMIC RADIATION AT SOLAR MINIMUM, 1965
}
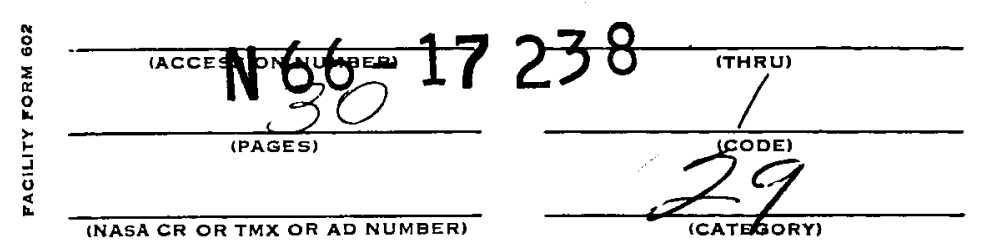

GPO PRICE

\$

CFSTI PRICE(S) \$

Hard copy $(\mathrm{HC})$

Microfiche (MF)

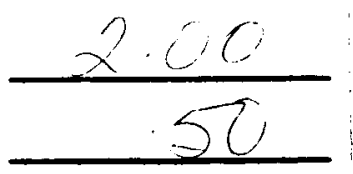
If 653 July 65

NOVEMBER 1965

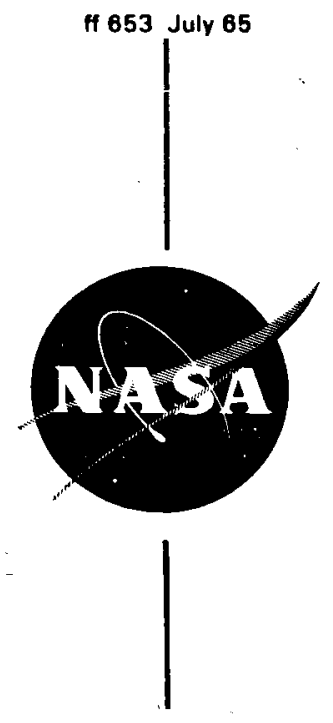

GODDARD SPACE FLIGHT CENTER GREENBELT, MARYLAND

Energetic Particles Preprint Series 


\section{THE MULTIPLY-CHARGED PRIMARY COSMIC RADIATION \\ AT SOLAR MINIMUM, 1965}

by
V. K. Balasubrahmanyan*
D. E. Hagge
G. H. Ludwig
F. B. MeDonald

November 1965

Goddard Space Flight Center

Greenbelt, Maryland

* On leave from Tata Institute of Fundamental Research, Bombay, India. 


\title{
THE MULTIPLY-CHARGED PRIMARY COSMIC RADIATION \\ AT SOLAR MINIMUM, 1965
}

\begin{abstract}
$N 6617238$

The primary cosmic ray charge and energy spectra have been obtained for Helium through Neon during the recent period when solar modulation effects were at a minimum. These spectra represent a synthesis of preliminary experimental results from cosmic ray experiments flown on OGO-I, IMP-III and high latitude Skyhook balloon flights. The He energy spectrum is given from 35 to 750 Mev/nucleon. The energy spectra for Lithium-Oxygen covers the interval $\sim 40-1200 \mathrm{Mev} /$ nucleon. Integral flux values $>1200 \mathrm{Mev}$ are also obtained for all components. L/M ratios of $0.29 \bullet .07$ at 100 $\mathrm{Mev} /$ nucleon and $0.30 \pm .03$ above $600 \mathrm{Mev} /$ nucleon are found. The $\mathrm{C} / \mathrm{He}$ ratio of $0.023 \pm .005$ at $100 \mathrm{Mev} /$ nucleon is significantly less than the same integral ratio of $0.036 \pm .004$ above $600 \mathrm{Mev} /$ nucleon, indicating energy dependent ionization and spallation losses during propagation through the interstellar gas under the assumption of similar source spectra.

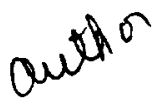




\section{CONTENTS}

Page

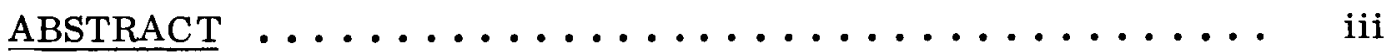

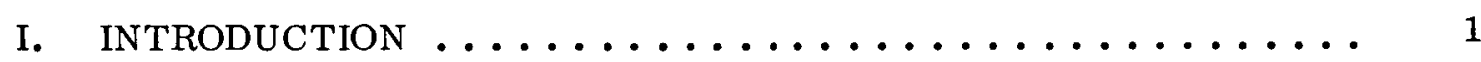

II. INSTRUMENTATION ....................... 2

A. $d E / d x$ vs $E \ldots \ldots \ldots \ldots \ldots \ldots \ldots \ldots \ldots \ldots \ldots \ldots \ldots \ldots \ldots \ldots \ldots \ldots$

B. Double $\mathrm{dE} / \mathrm{dx}-$ Cerenkov Counter Telescope ........... 4

III. DATA ANALYSIS AND RESULTS ................. 5

IV. DISCUSSION ............................ 7

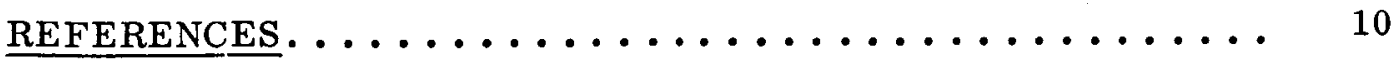




\section{LIST OF ILLUSTRATIONS}

Page

Figure 1(a) Schematic drawing of $\mathrm{dE} / \mathrm{dx}$ vs $\mathrm{E}$ detector used on IMP and OGO-I ..................... 12

Figure 1(b) Schematic drawing of Cerenkov-double $\mathrm{dE} / \mathrm{dx}$ telescope used on high latitude balloon flights. The two measurements of $\mathrm{dE} / \mathrm{dx}$ are very effective in eliminating background due to nuclear interactions. The Cerenkov counter has a threshold of $330 \mathrm{Mev} /$ nucleon. The top surface of this sensor is blackened to eliminate upward moving albedo ............................ 12

Figure 2 Mass and energy response of $d E / d x$ vs $E$ telescope $(\triangle \mathrm{E}$ proportional to $\mathrm{dE} / \mathrm{dx} ; \mathrm{E}$ determined from

E - $\Delta \mathrm{E}$ measurement in lower scintillator) $\ldots \ldots \ldots . . . .13$

Figure 3 Raw data from the OGO-I dE/dx vs E high gain mode. Helium is well resolved (see Figure 5 for mass histogram) .................. 14

Figure 4 Raw data from the OGO-I dE/dx vs E low gain mode.... 15

Figure 5 Mass histogram for 5 energy intervals in helium region. Asymmetry in lower channels is due to presence of $\mathrm{He}^{3} \ldots \ldots \ldots \ldots$ 
Figure $6 \quad$ Charge spectrum at the high energies as obtained by a Skyhook balloon flight at Ft. Churchill on June 12, 1965 using a Cerenkovdouble $\mathrm{dE} / \mathrm{dx}$ telescope. The end points of the individual lines represent the two measurements of ionization loss. A selection criteria has been applied which demands the two measurements be within $\pm 25 \%$ of each other. An appropriate correction is made for Landau fluctuations $\ldots \ldots \ldots \ldots \ldots$

Figure 7 The composite energy spectra of nuclei from protons to oxygen from $25 \mathrm{Mev} /$ nucleon to

$1 \mathrm{Bev} /$ nucleon. The proton spectrum is from IMP III ......................... 18

Figure 8 Calculated variation of the ratio of

$\left(\mathrm{dJ} / \mathrm{dE}_{\text {carbon }}\right)_{100 \text { Mev/nucleon }} /\left(\mathrm{dJ} / \mathrm{dE}_{\text {hel ium }}\right)_{100 \text { Mev/nucleon }}$ to $(\mathrm{dJ} / \mathrm{dE}$ carbon $)>600$ Mev/nucleon $/\left(\mathrm{dJ} / \mathrm{dE}_{\text {hel ium }}\right)>600$ Mev/nucleon plotted as a function of the interstellar matter traversed. The calculation allows for ionization loss of the nuclei in the interstellar medium but neglects nuclear interactions . . . . . . 19 Figure 9 Summary of L/M ratios. Arrow denotes integral measurement ................. 20 


\section{THE MULTIPLY-CHARGED PRIMARY COSMIC RADIATION}

AT SOLAR MINIMUM, 1965

\section{INTRODUCTION}

This paper summarizes the preliminary results obtained from cosmic ray experiments flown on OGO-I, IMP-III, and Fort Churchill Skyhook balloon flights. A synthesis of these studies provides charge and energy spectra of the galactic cosmic rays extending from helium through neon with differential energy measurements, typically from about $25 \mathrm{Mev} /$ nucleon to $1 \mathrm{Bev} /$ nucleon. Particular emphasis is placed on the period from March through June of 1965 when the effects of solar modulation appear to be at a mimimum.

The low energy spectra and charge composition are of special interest since these features should be most sensitive to the three basic processes which influence the galactic cosmic rays before reaching the vicinity of the earth. These processes are:

1. Initial acceleration and escape from the source region,

2. Diffusion through the galaxy with possible further acceleration, and

3. Solar modulation.

The hydrogen and helium spectra are used to study the effects of solar modulation and will be discussed in a later paper. The ratio of the elements from helium through neon should not be affected by solar modulation and should, 
therefore, provide insight into the mechanism and conditions of initial acceleration and propagation through the galaxy.

\section{INSTRUMENTATION}

Two detector systems are used: $a \mathrm{dE} / \mathrm{dx}$ vs $\mathrm{E}$ scintillation-counter telescope and a Cerenkov-double $\mathrm{dE} / \mathrm{dx}$ scintillation-counter telescope. The properties of these devices are summarized in Table $\mathrm{I}$.

Table I

GSFC Detector Systems Used to Study Galactic

Cosmic Rays at Solar Minimum, 1965

\begin{tabular}{|c|c|c|c|}
\hline Satellite & $\begin{array}{l}\text { Apogee in } \\
\text { Earth Radii }\end{array}$ & $\begin{array}{c}\text { Principal Data } \\
\text { Coverage }\end{array}$ & $\begin{array}{c}\text { Goddard Cosmic } \\
\text { Ray Instrumentation }\end{array}$ \\
\hline OGO-I & $24.3 \mathrm{Re}_{\mathrm{e}}$ & Sept., 1964 to June, 1965 & $\begin{array}{l}\text { dE/dx vs E covering } \\
\text { Hydrogen through } \\
\text { Neon; Geiger Counter } \\
\text { Monitor (three } \\
\text { Telescopes) }\end{array}$ \\
\hline IMP III & $41.5 \mathrm{R}_{\mathrm{e}}$ & May, 1965 to June, 1965 & $\begin{array}{l}\mathrm{dE} / \mathrm{dx} \text { vs } \mathrm{E} \text { covering } \\
\mathrm{e}, \mathrm{H}, \text { and He; Geiger } \\
\text { Counter Monitor (two } \\
\text { telescopes) }\end{array}$ \\
\hline $\begin{array}{l}\text { Skyhook } \\
\text { Balloons }\end{array}$ & $3 \mathrm{gm} / \mathrm{cm}^{2}$ & June 1965 & $\begin{array}{l}\text { Cerenkov-double } \\
(\mathrm{dE} / \mathrm{dx})\end{array}$ \\
\hline
\end{tabular}




\section{A. $\mathrm{dE} / \mathrm{dx}$ vs $\mathrm{E}$}

The $\mathrm{dE} / \mathrm{dx}$ vs $\mathrm{E}$ telescope (Figure 1a) is an arrangement of two scintillators to give both the total energy $\mathrm{E}$ of a charged particle and its rate of energy loss $\mathrm{dE} / \mathrm{dx}$ (Bryant et al, 1962). A CsI(T1) crystal of $0.45 \mathrm{gm} / \mathrm{cm}^{2}(1 \mathrm{~mm})$ thickness absorbs energy $\triangle \mathrm{E}$ which is proportional to the $\mathrm{dE} / \mathrm{dx}$ of the particle. The second CsI(T1) crystal of $9 \mathrm{gm} / \mathrm{cm}^{2}(2 \mathrm{~cm})$ thickness absorbs the remaining $\mathrm{E}-\Delta \mathrm{E}$ whenever a particle of energy E comes to rest in that crystal as determined by the additional thin $\left(0.8 \mathrm{gm} / \mathrm{c} \mathrm{m}^{2}\right)$ plastic guard scintillator operated in anticoincidence. The outputs of the $\triangle \mathrm{E}$ and $\mathrm{E}-\triangle \mathrm{E}$ scintillators are analyzed only when light is observed from the CsI crystals but not from the guard scintillator. Thus, this detector is sensitive to particles with energies high enough to penetrate the $\Delta \mathrm{E}$ crystal, yet low enough to be stopped in the $\mathrm{E}-\Delta \mathrm{E}$ scintillator. For $\mathrm{H}$ and $\mathrm{He}$, this energy range is from 15 to $80 \mathrm{Mev} /$ nucleon. This range changes for heavier nuclei, e.g., for carbon it is 25 to $145 \mathrm{Mev} /$ nucleon. Excellent resolution can also be obtained for low energy electrons in the $3-12 \mathrm{Mev}$ range on the IMP package as has been discussed elsewhere (Cline et al., 1964).

The two measurements made on each particle stopping in the telescope provide both charge and mass resolution (Figure 2). On the IMP satellite two 512-channel differential pulse height converters are used and electron, hydrogen, and the helium regions are covered. On the OGO satellite gain changing devices permit coverage of nuclei from hydrogen through neon.

On the OGO-I satellite a boom which was folded across the instrument during launch failed to deploy and added about $.5 \mathrm{gm} / \mathrm{cm}^{2}$ of aluminum shielding within a portion of the solid angle. Because of the close proximity of this shielding to 
the detector significant angular divergence of the spallation products does not occur; thus most of the spallation products from a given interaction increase the background only slightly in the region of $\mathrm{He}$ and above.

This telescope provides a means of studying the low energy component in the presence of higher energy cosmic rays. To illustrate the resolution, raw data typical of both high and low gain modes of operation of OGO are shown in Figure 3 and 4 respectively. Not only is the He clearly separated from the background but the resolution of $\mathrm{He}^{3}$ and $\mathrm{He}^{4}$ is observable. The He mass histograms for five energy intervals from IMP are shown in Figure 5. These are obtained by summing the data channels constructed parallel to and centered about the $\mathrm{He}^{4}$ line of a plot similar to that of Figure 3. In Figure 4, the separation of the heavier particles from the background is also apparent.

\section{B. Double $\mathrm{dE} / \mathrm{dx}$ - Cerenkov Counter Telescope}

Although this detector has been flown on Skyhook balloon flights in June of 1963, 1964 and 1965, only the 1965 results will be discussed here. In each flight a floating altitude of $3 \mathrm{gm} / \mathrm{cm}^{2}$ residual atmosphere was reached, greatly reducing the correction for secondaries. A schematic drawing of the detector is shown in Figure 1b. The system is similar in principle to that reported by McDonald and Webber (1962) except for some technical details to achieve better charge and energy resolution. For each particle traversing the telescope the pulse heights from all three sensors are recorded. For each sensor there is a 512 channel pulse height analyzer; the data are recorded on a balloon borne tape recorder. Gain switching permits coverage of the energy range from hydrogen through neon. Differential energy spectra are obtained for $B$ through $O$ in the region from 600 
to $1200 \mathrm{Mev} /$ nucleon and integral flux values are obtained for each charge component above $600 \mathrm{Mev} /$ nucleon. The energy spectra for helium from 125 to 800 Mev/nucleon are deduced.

Figure 6 illustrates the excellent charge resolution for light and medium nuclei from the Cerenkov-scintillator telescope flown at $3 \mathrm{gm} / \mathrm{cm}^{2}$ in a balloon at Ft. Churchill on June 12, 1965. The predominance of $\mathrm{C}$ and $\mathrm{O}$, the paucity of light elements, and the low abundance of $\mathrm{N}$ and $\mathrm{F}$ are apparent.

\section{DATA ANALYSIS AND RESULTS}

The format illustrated for the OGO-I telescope in Figures 3 and 4 is the printout of a 7094 computer program displaying the pulse height analyzer outputs in two dimensions. The analyses performed on these data are a statistical biterror removal of noisy data and a correction for photomultiplier gain changes. The isotopes of $\mathrm{He}^{3}$ and $\mathrm{He}^{4}$ and all charge groups through Ne are well resolved. The end-point energies depend upon the particle range only and therefore provide instrument calibration. The energies of the end-points vary from $80 \mathrm{Mev} / \mathrm{nucleon}$ for He up to $170 \mathrm{Mev} /$ mucleon for 0 . It is apparent from the data that $\mathrm{C}$ and $\mathrm{O}$ are the most abundant elements at these low energies. There is a very low abundance of $\mathrm{Li}, \mathrm{Be}$, and $\mathrm{N}$, relatively more $\mathrm{B}$ and no measurable $\mathrm{F}$.

In Figure 6 the pulse heights from the two scintillators and the Cerenkov counter of the balloon experiment are shown. The end points of the individual lines represent the two measurements of ionization loss. A selection criterion has been applied which demands that the two measurements are within $25 \%$ of each other. An appropriate correction is made for Landau fluctuations. 
The energy spectra of the nuclei from $\mathrm{H}$ through $\mathrm{O}$ between $25 \mathrm{Mev} /$ nucleon and $1 \mathrm{Bev} /$ nucleon are shown in Figure 7. It is seen that the energy spectra of all components are quite flat in the region of a few hundred $\mathrm{Mev} / \mathrm{nucleon}$ at solar minimum. The charge composition relative to carbon in the two energy ranges is given in Table II.

Table II

Relative Nuclear Abundances

\begin{tabular}{|c|c|c|}
\hline Elements & Energy $=100 \mathrm{Mev} / \mathrm{nuc}$ & $\begin{array}{c}\text { Energy }>600 \mathrm{Mev} / \mathrm{nuc} \\
\text { at } \sim 3 \mathrm{gms} / \mathrm{cm}^{2} \text { of atmosphere }\end{array}$ \\
\hline $\mathbf{L i}$ & 0.15 & 0.3 \\
\hline $\mathrm{Be}$ & 0.09 & 0.2 \\
\hline B & 0.38 & 0.4 \\
\hline $\mathrm{C}$ & 1.0 & 1.0 \\
\hline $\mathrm{N}$ & 0.18 & 0.3 \\
\hline $\mathrm{O}$ & 0.84 & 0.8 \\
\hline $\mathbf{F}$ & $<0.03$ & 0.04 \\
\hline $\mathrm{Ne}$ & & 0.14 \\
\hline \multicolumn{3}{|c|}{ Top of Atmosphere } \\
\hline $\mathrm{L} / \mathrm{M}$ & $0.29 \pm .07$ & $0.30 \pm .03$ \\
\hline $\mathrm{C} / \mathrm{He}$ & $0.023 \pm .005$ & $0.036 \pm .004$ \\
\hline
\end{tabular}

The $\mathrm{L} / \mathrm{M}$ ratio for energies greater than $600 \mathrm{Mev} /$ nucleon is found from the balloon measurement at the top of the atmosphere using fragmentation parameters of Friedlander et al. (1963). This ratio corrected to the top of the 
atmosphere, is found to be $0.30 \pm .03$. At $100 \mathrm{Mev} /$ nucleon the $\mathrm{L} / \mathrm{M}$ ratio is $0.29 \pm .07$, in agreement with that found at high energies.

The $\mathrm{C} / \mathrm{He}$ ratio for energies $>600 \mathrm{Mev} /$ nucleon is found to be $0.036 \pm .004$ whereas at $100 \mathrm{Mev} /$ nucleon the value is $0.023 \pm .005$.

\section{DISCUSSION}

The decrease in the ratio of $\mathrm{C} / \mathrm{He}$ with a decrease in energy agrees qualitatively with the explanation of Fichtel et al. (1965) that nuclei of higher $\mathrm{Z}$ undergo larger ionization losses per nucleon in the interstellar medium. This tends to deplete the nuclei of high $\mathrm{Z}$ compared to those of low $\mathrm{Z}$ at low energies, assuming that they have similar source spectra. These conclusions are independent of velocity or rigidity dependent solar modulation effects.

Figure 8 shows the calculated ratio of the carbon and helium nuclei fluxes at $100 \mathrm{Mev} /$ nucleon to that at $600 \mathrm{Mev} /$ nucleon for different amounts of interstellar matter traversed, assuming source spectra of the following types:

1. Flat differential distribution, i.e. $d N / d E "=1$

2. A spectrum of the form const/(1+E) ${ }^{2.5}$

3. A spectrum of the form const/ $E^{2.5}$, where $E$ is the kinetic energy/nucleon in Bev.

The thick line at the center of Figure 8 represents the experimental ratio, and the dotted lines on either side show the limits of the error. In these calculations only ionization loss has been considered, and effects of the energy dependence of fragmentation parameters are ignored. Within the limitations of the 
experimental ratio it is hard to choose between spectra of form 2 or 3 , and determine the matter traversed based on this ratio alone. Since we find the $\mathrm{L} / \mathrm{M}$ ratios at both 100 and $600 \mathrm{Mev} /$ nucleon are equal, the fragmentation contributions may also be taken as approximately equal. Then the change in the $\mathrm{C} / \mathrm{He}$ ratio with energy can be interpreted as due to ionization losses alone and the data seem to suggest the low energy source flux increases more rapidly then $\mathrm{K} /(1+\mathrm{E})^{2 \cdot 5}$

In Table III and Figure 9 a number of the most recent measurements of the $\mathrm{L} / \mathrm{M}$ ratio are shown along with those from this paper. Our measurement above $600 \mathrm{Mev} /$ nucleon is in good agreement with all others (Balasubrahmanyan and McDonald, 1964; Badhwar et al., 1965; Koshiba et al., 1963; Foster and Debenedetti, 1963; O'Dell et al, 1961; and Aizu et al., 1962) in this energy range. The measurement at $100 \mathrm{Mev} /$ nucleon obtained from the OGO-I satellite, which requires no fragmentation and energy loss corrections, is in agreement with measurements above $600 \mathrm{Mev} /$ nucleon also. The results of emulsion experiments (Badhwar et al., 1965; Koshiba et al., 1963; Foster and Bedenetti, 1963; and Aizu et al., 1962) in the 200-700 Mev/nucleon range require a sharp increase in the $\mathrm{L} / \mathrm{M}$ ratio. This would imply strong energy dependence of fragmentation parameters, since again this ratio should be relatively independent of solar modulation.

These results do indicate a preferential slowing down of $\mathrm{M}$ nuclei relative to $\mathrm{He}$, as opposed to acceleration in the interstellar medium. In order to understand better the acceleration and propagation of cosmic rays, it will be necessary to know and account for the energy dependence of fragmentation parameters and 
ionization losses in any interpretation of measurements of the energy spectra of the primary components in this region. More extensive data analysis on OGO-I data considering heavier components is in progress. This should improve the situation in the $100 \mathrm{Mev} /$ nucleon range. More detailed studies of the charge and energy spectra below $700 \mathrm{mev} /$ nucleon seem very important.

Table III

Summary of L/M Values

\begin{tabular}{|c|c|c|c|}
\hline $\begin{array}{c}\text { Energy } \\
\text { (īMev/nucleon) }\end{array}$ & $\mathbf{L} / \mathbf{M I}$ & Time of Mcasurcment & Source \\
\hline 100 & $0.29 \pm .07$ & March-June 1965 & This paper, satellite \\
\hline$>600$ & $0.30 \pm .03$ & June 1965 & $\begin{array}{l}\text { This paper, } 2.8 \\
\mathrm{gms} / \mathrm{cm}^{2}\end{array}$ \\
\hline $\begin{array}{l}400-800 \\
>800\end{array}$ & $\begin{array}{l}0.28 \pm .08 \\
0.28 \pm .08\end{array}$ & June 1963 & $\begin{array}{l}\text { Balasubrahmanyan \& } \\
\text { McDonald, } 5 \mathrm{gm} / \mathrm{cm}^{2}\end{array}$ \\
\hline $\begin{array}{l}200-700 \\
>700\end{array}$ & $\begin{array}{l}* 0.53 \pm .11 \\
* 0.25 \pm .04\end{array}$ & August 1962 & $\begin{array}{l}\text { Badhwar et al } \\
4.7 \mathrm{gms} / \mathrm{cm}^{2}\end{array}$ \\
\hline $\begin{array}{l}200-700 \\
>700\end{array}$ & $\begin{array}{l}0.51 \pm .07 \\
0.32 \pm .03\end{array}$ & September 1959 & $\begin{array}{l}\text { Koshiba et al } \\
1.5-2.5 \mathrm{gms} / \mathrm{cm}^{2}\end{array}$ \\
\hline $\begin{array}{l}200-1000 \\
>1500\end{array}$ & $\begin{array}{l}0.43 \pm .07 \\
0.28 \pm .05\end{array}$ & August 1958 & $\begin{array}{l}\text { Foster \& Debenedetti } \\
3.8 \mathrm{gms} / \mathrm{cm}^{2}\end{array}$ \\
\hline$>1500$ & $0.25 \pm .06$ & March 1958 & $\begin{array}{l}\text { O'Dell et al, } 2.7 \\
\text { gms/cm }\end{array}$ \\
\hline $\begin{array}{l}200-700 \\
>700\end{array}$ & $\begin{array}{l}0.37 \pm .05 \\
0.21 \pm .03\end{array}$ & September 1957 & $\begin{array}{l}\text { Aizu et al } \\
8.0 \mathrm{gms} / \mathrm{cm}^{2}\end{array}$ \\
\hline
\end{tabular}

${ }^{*}$ Calculated from $\mathrm{L} / \mathrm{S}$ value using $\mathrm{H} / \mathrm{M}=0.30 \pm .02$ (Weber, W. R., 1964). 


\section{$\underline{\text { References }}$}

Aizu, H., E. Tamai, M. Koshiba, and E. Lohrmann, Proc. of Int. Conf. on Cosmic Rays, Kyoto, 1962.

Badhwar, G. D., S. N. Devanathan and M. F. Kaplon, The Energy Dependence of the Abundance of Lithium, Berylliym and Boron in the Primary Cosmic Radiation, J. Geophys. Res., 70, 1005, 1965.

Balasubrahmanyan, V. K. and F. B. McDonald, Solar Modulation Effects on the Primary Cosmic Radiation Near Solar Minimum, J. Geophys. Res., 69, (15), 3289, 1964.

Bryant, D. A., G. H. Ludwig, and F. B. McDonald, A Scintillation-Counter Telescope For Charge and Mass Identification of Primary Cosmic Rays, I.R.E. Trans. on N. S., Vol. N.S.-9, June 1962.

Cline, T. L., G. H. Ludwig, and F. B. McDonald, Detection of Interplanetary 3- to 12-Mev Electrons, Phys. Rev. Letters, Dec. 28, 1964.

Fichtel, C. E., D. E. Guss, and K. A. Neelakantan, An Experimental Examination of Low Energy Cosmic Ray Heavy Nuclei, Phys. Rev. 138, B732, 1965.

Foster, F. and A. Debenedetti, I1 Nuovo Cimento XXVIII, 1190, 1963.

Friedlander, M. W., K. A. Neelakantan, S. Tokunaga, G. R. Stevenson, and C. J. Waddington, 1963, Phili Mag., $\underline{8}, 1961$.

Koshiba, M., E. Lohrmann, H. Aizu, and E. Tamai, Nuclei of the Primary Cosmic Radiation with $\mathrm{Z} \geq 2$ at High Latitudes, Phys. Rev., $\underline{131}, 2692,1963$.

McDonald, F. B. and W. R. Webber, Cerenkov Scintillation Counter Measurements of the Light, Medium and Heavy Nuclei in the Primary Cosmic Radiation from Sunspot Minimum to Sunspot Maximum, J. Geophys, Res., 67, (6), 2119, 1962. 
O'Dell, F. W., M. M. Shapiro, and B. Stiller, Relative Abundance of the Heavy Nuclei of the Galactic Cosmic Radiation, Proc. Kyoto Conf. Cosmic Rays, 1961.

Webber, W. R., 1964 Univ. of Minn. Technical Report CR-76. 

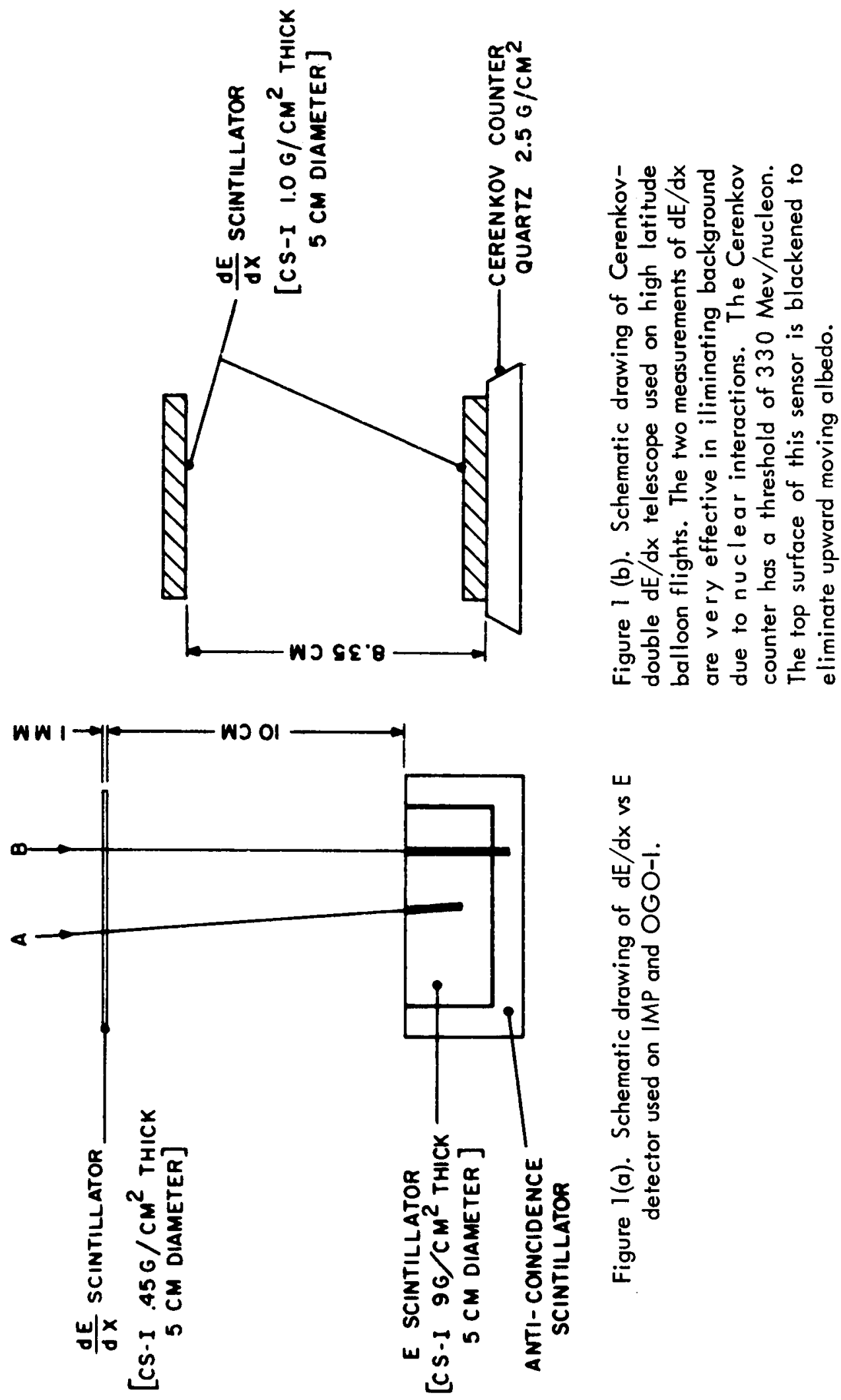


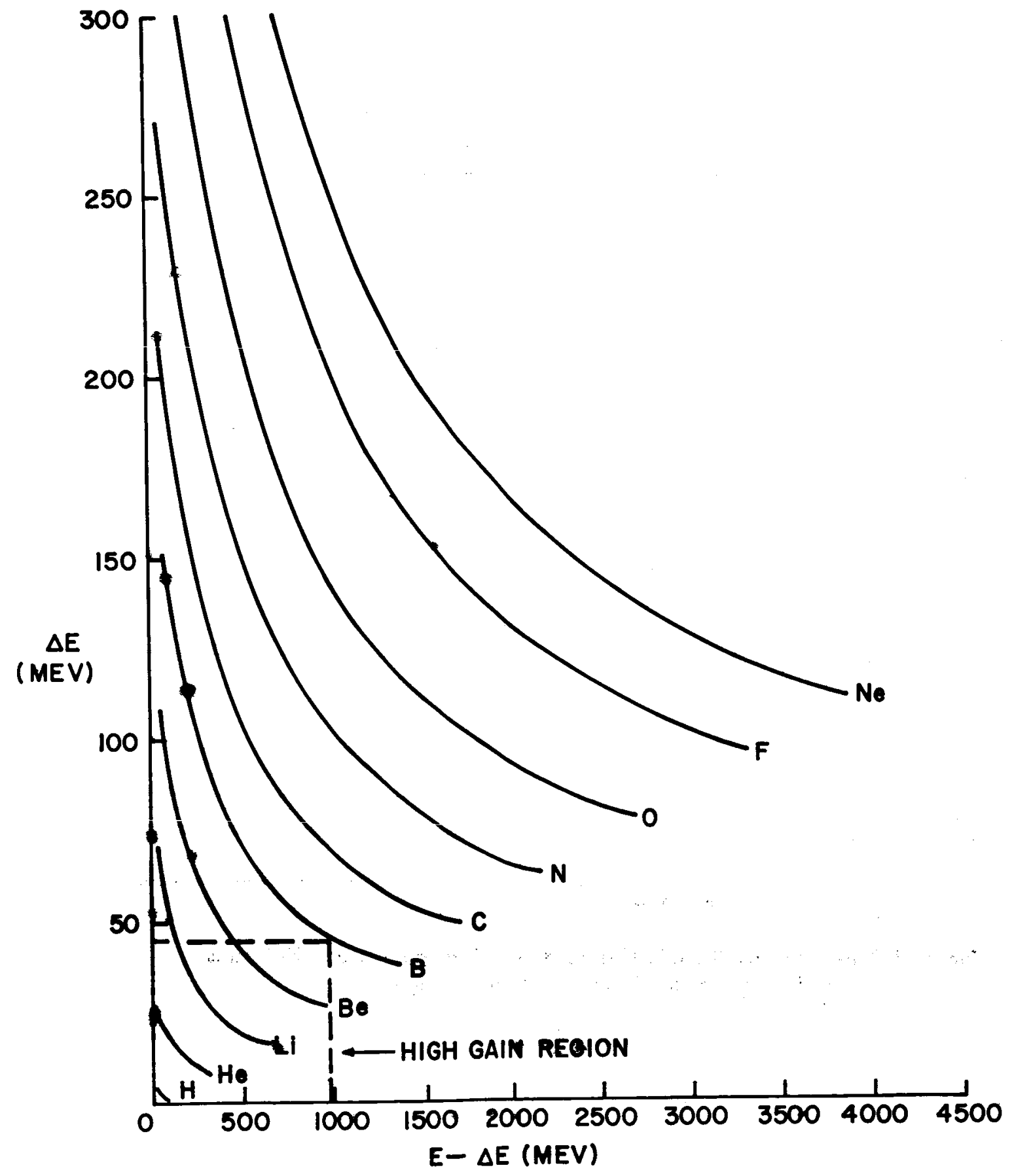

Figure 2. Mass and energy response of $d E / d x$ vs $E$ telescope ( $D E$ proportional to $d E / d x$; $E$ determined from $E-\triangle E$ measurement in lower scintillator). 


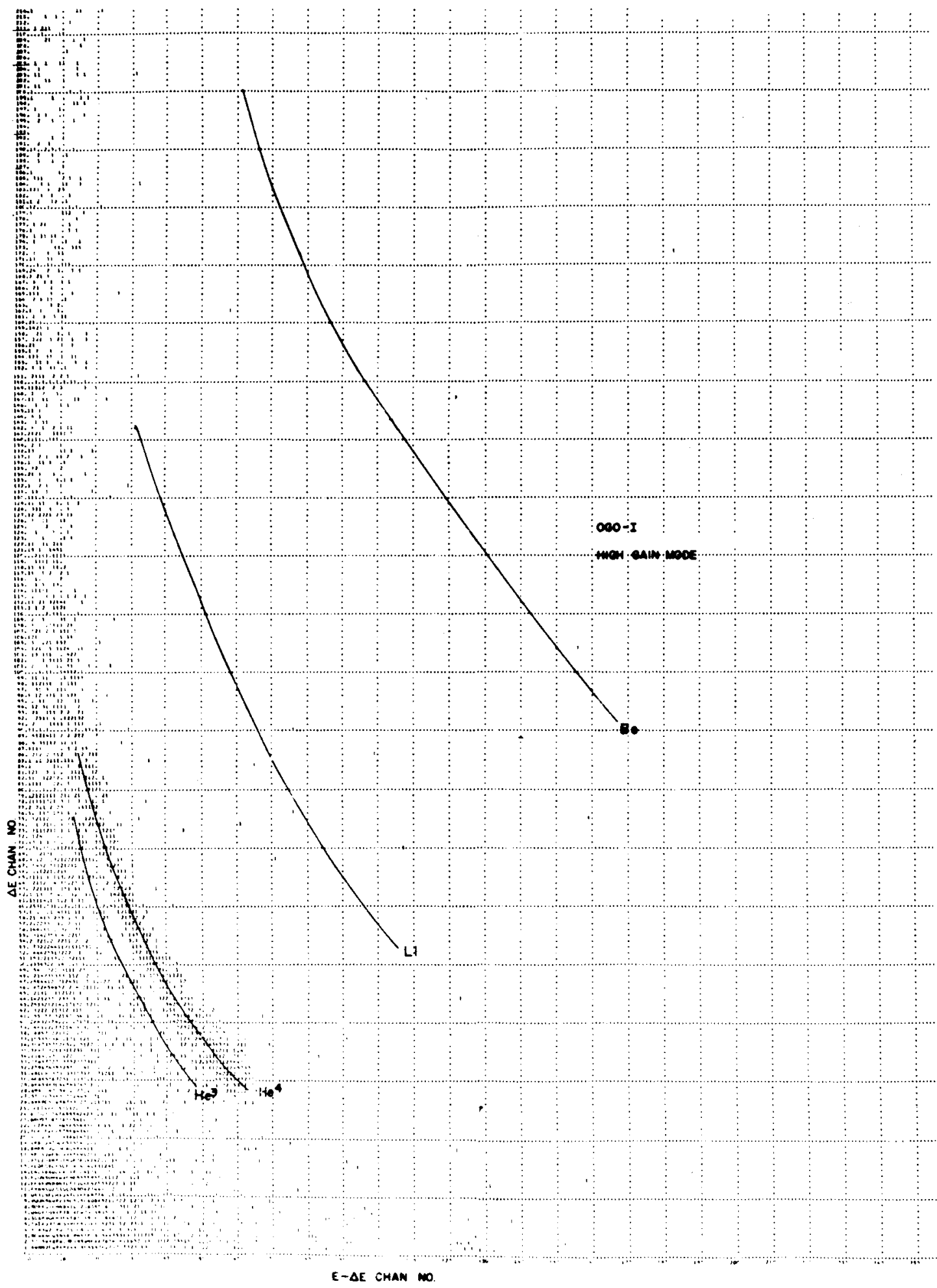

Figure 3. Raw data from the OIGO-I dE/dx vs $E$ high gain mode. Helium is well resolved (see Figure 5 for mass histogram). 


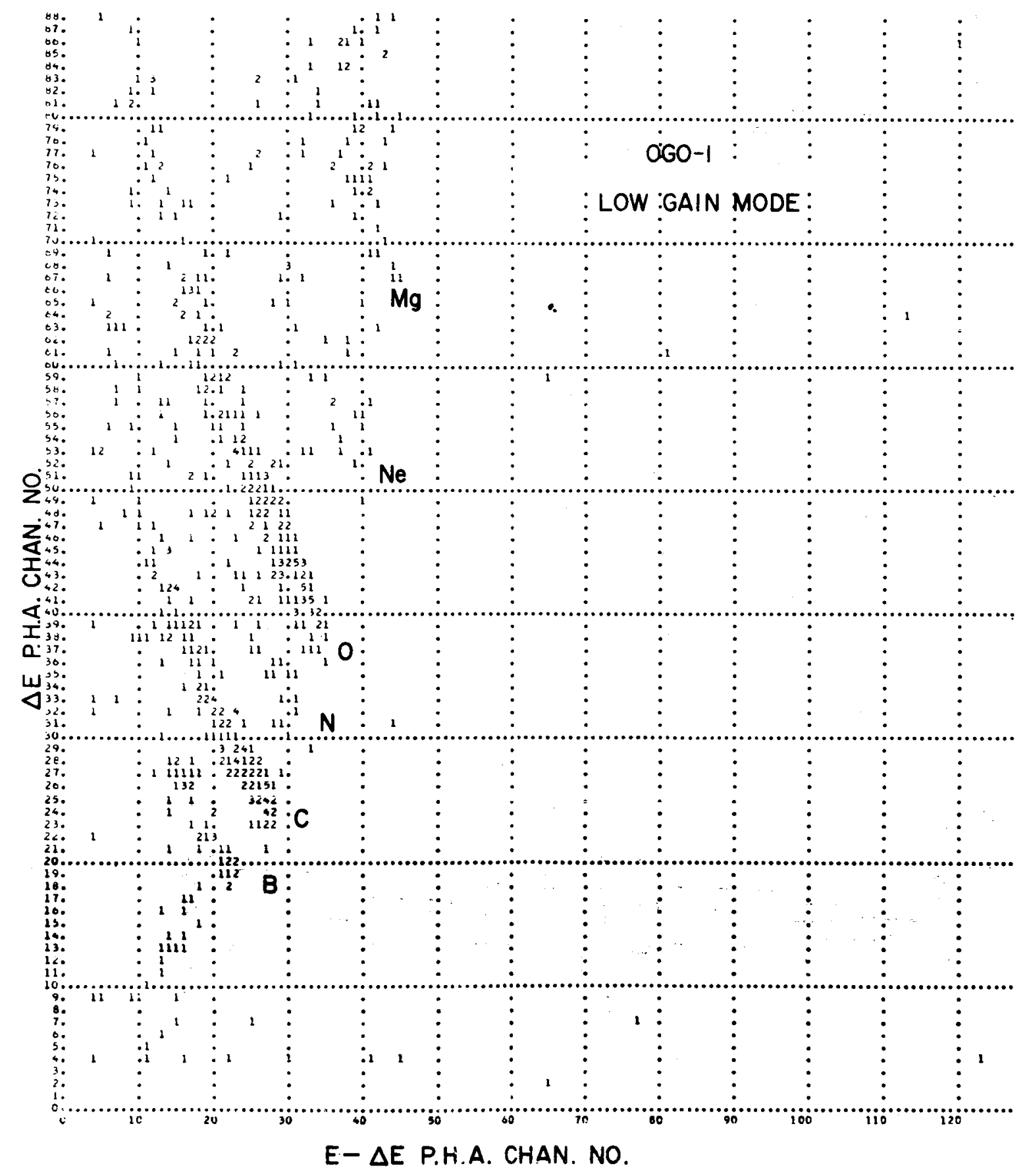

Figure 4. Raw data from the $O G O-I d E / d x$ vs $E$ low gain mode. 

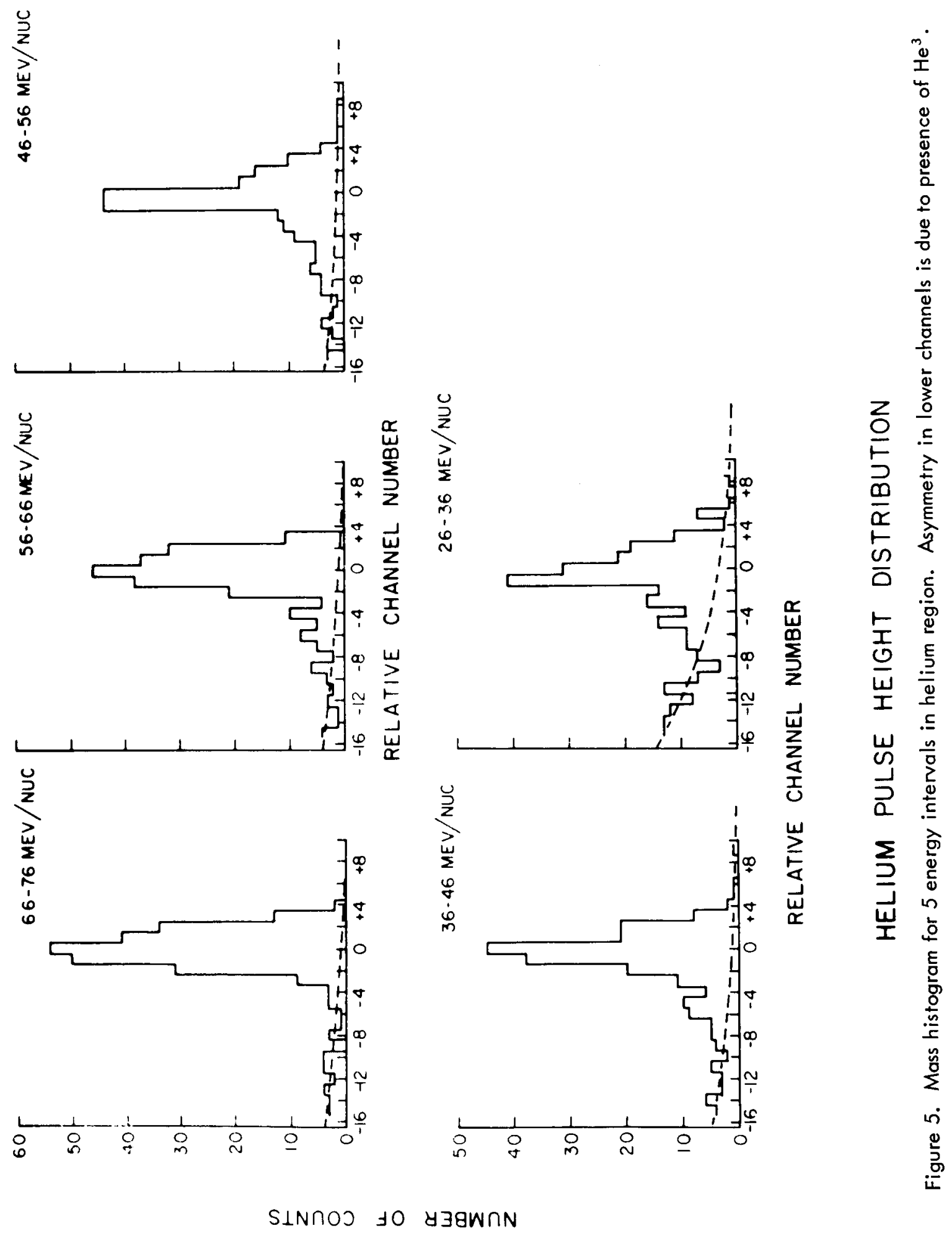


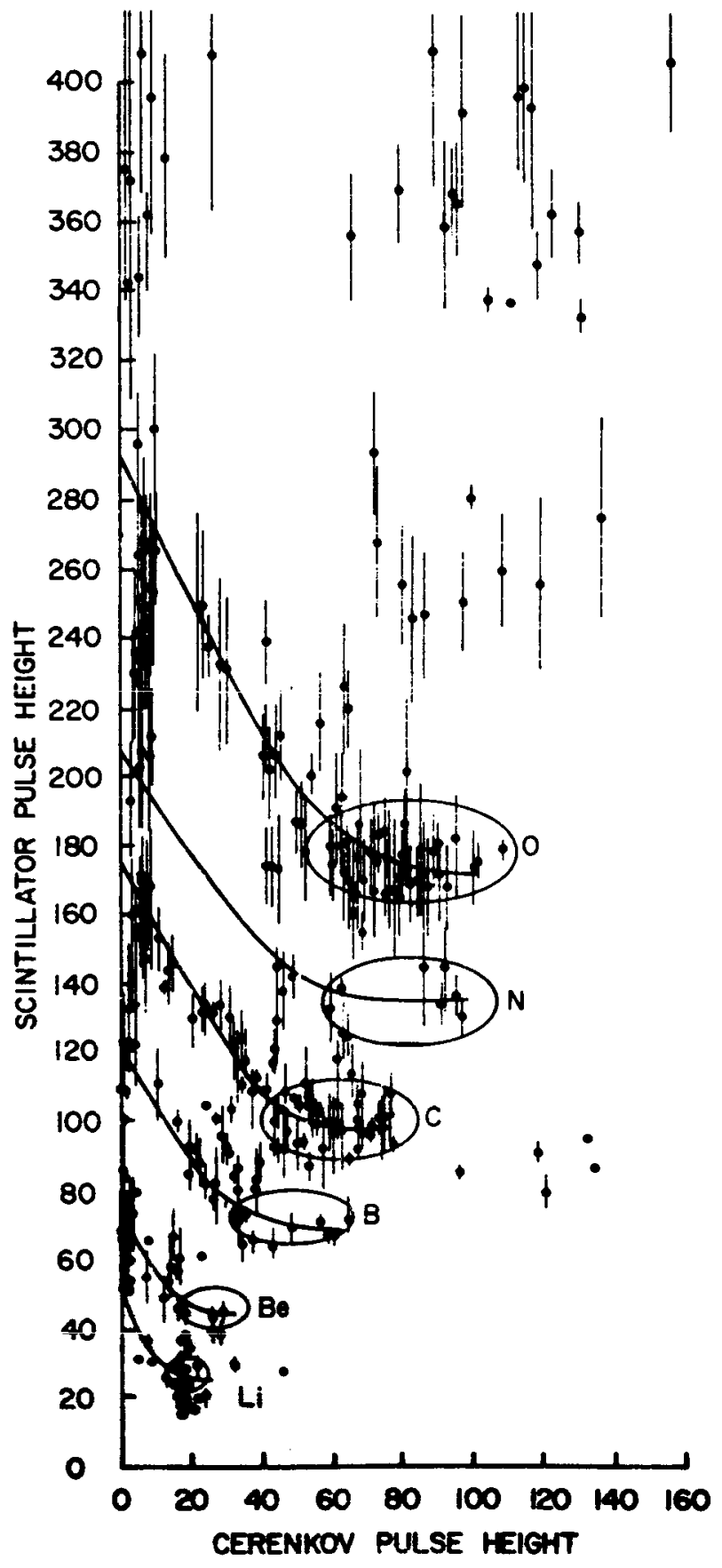

Figure 6. Charge spectrum at the high energies as ob.tained by a Skyhook balloon flight at Ft. Churchill on June 12, 1965 using a Cerenkovdouble $d E / d x$ telescope. The end points of the individual lines represent the two measurements of ionization loss. A selection criteria has been applied which demands the two measurements be within $\pm 25 \%$ of each other. An appropriate correction is made for Landau fluctuations. 


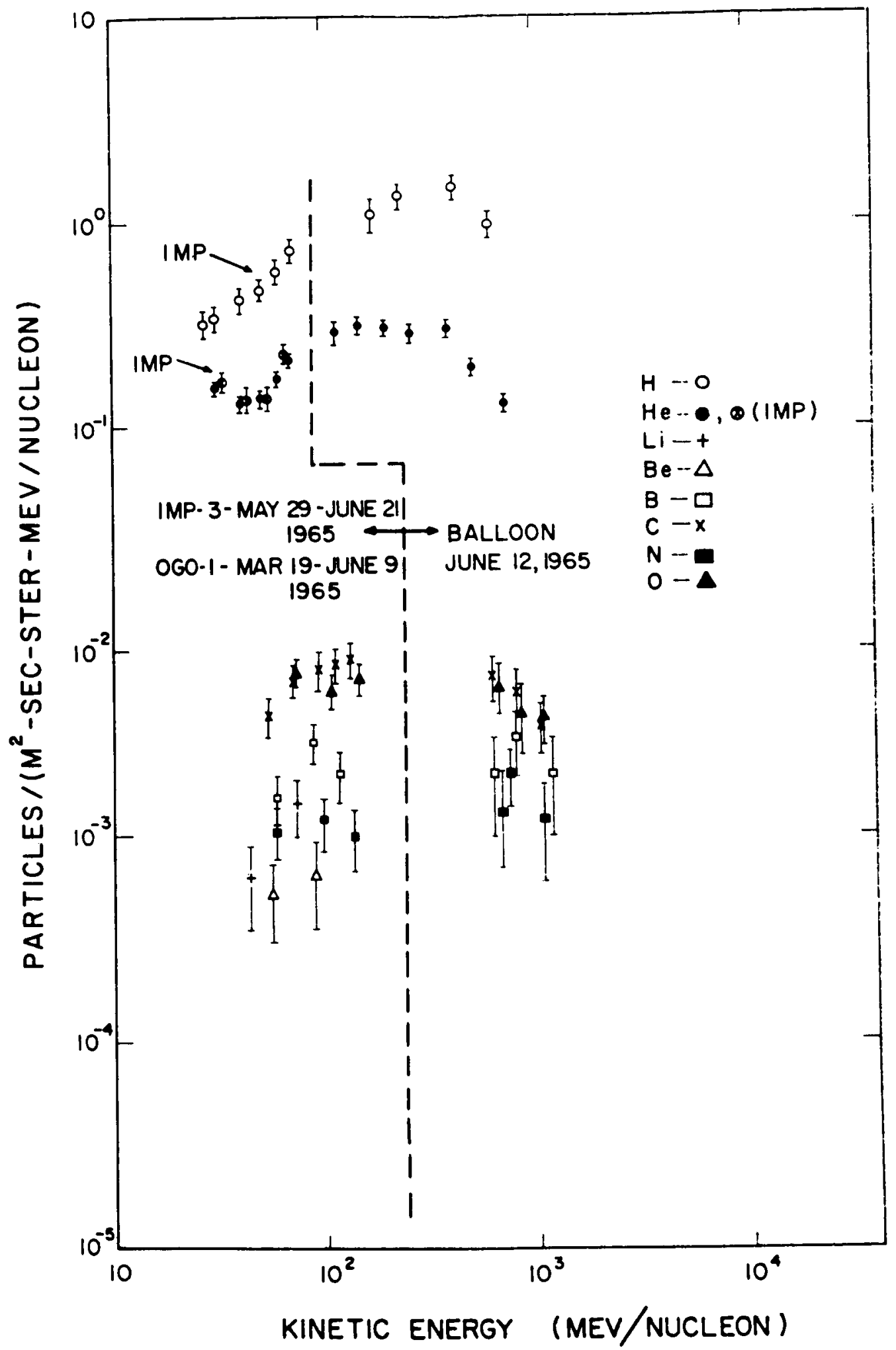

Figure 7. The composite energy spectra of nuclei from protons to oxygen from $25 \mathrm{Mev} /$ nucleon to $1 \mathrm{Bev} /$ nucleon. The proton spectrum is from IMP III. 


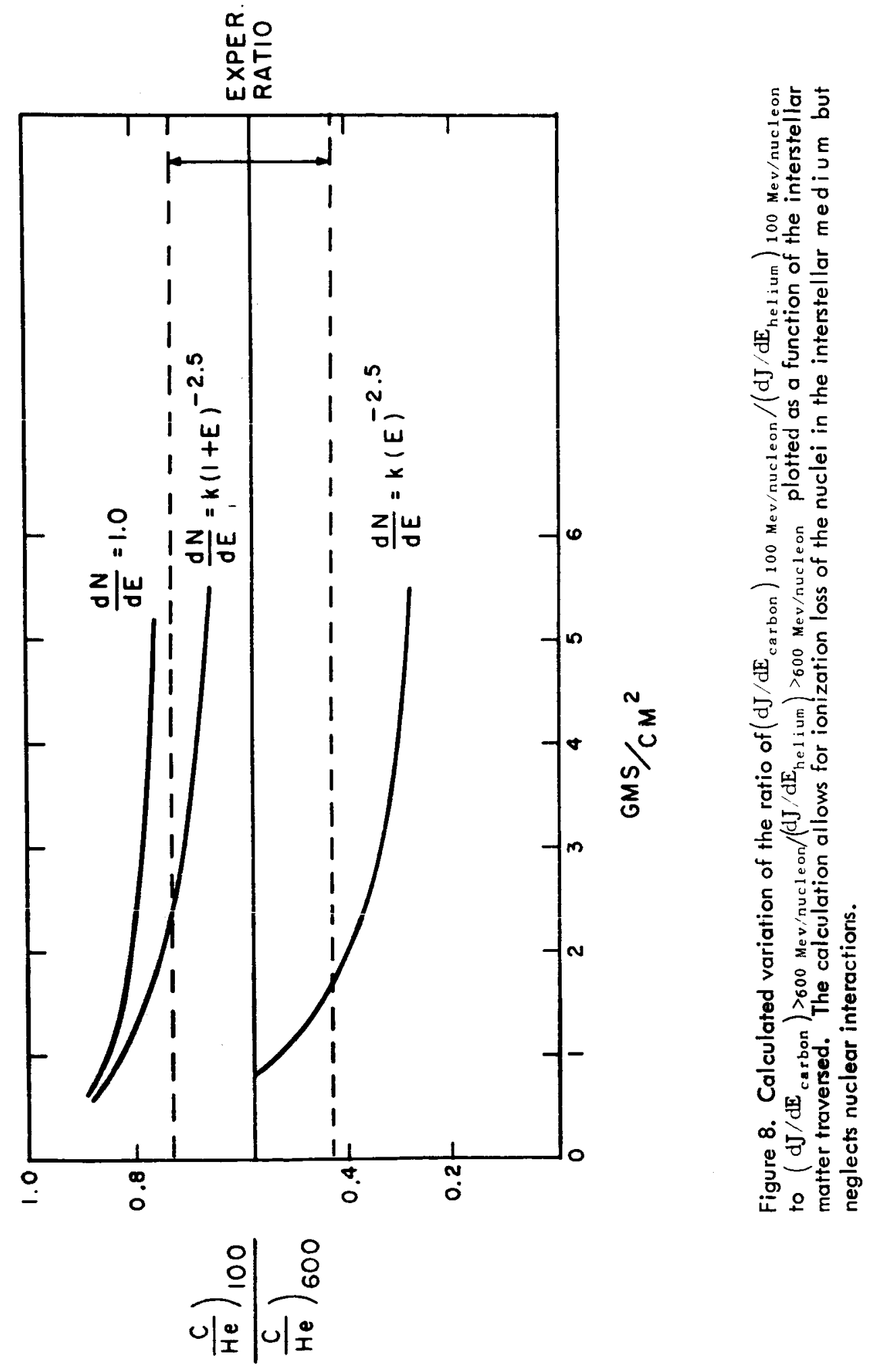




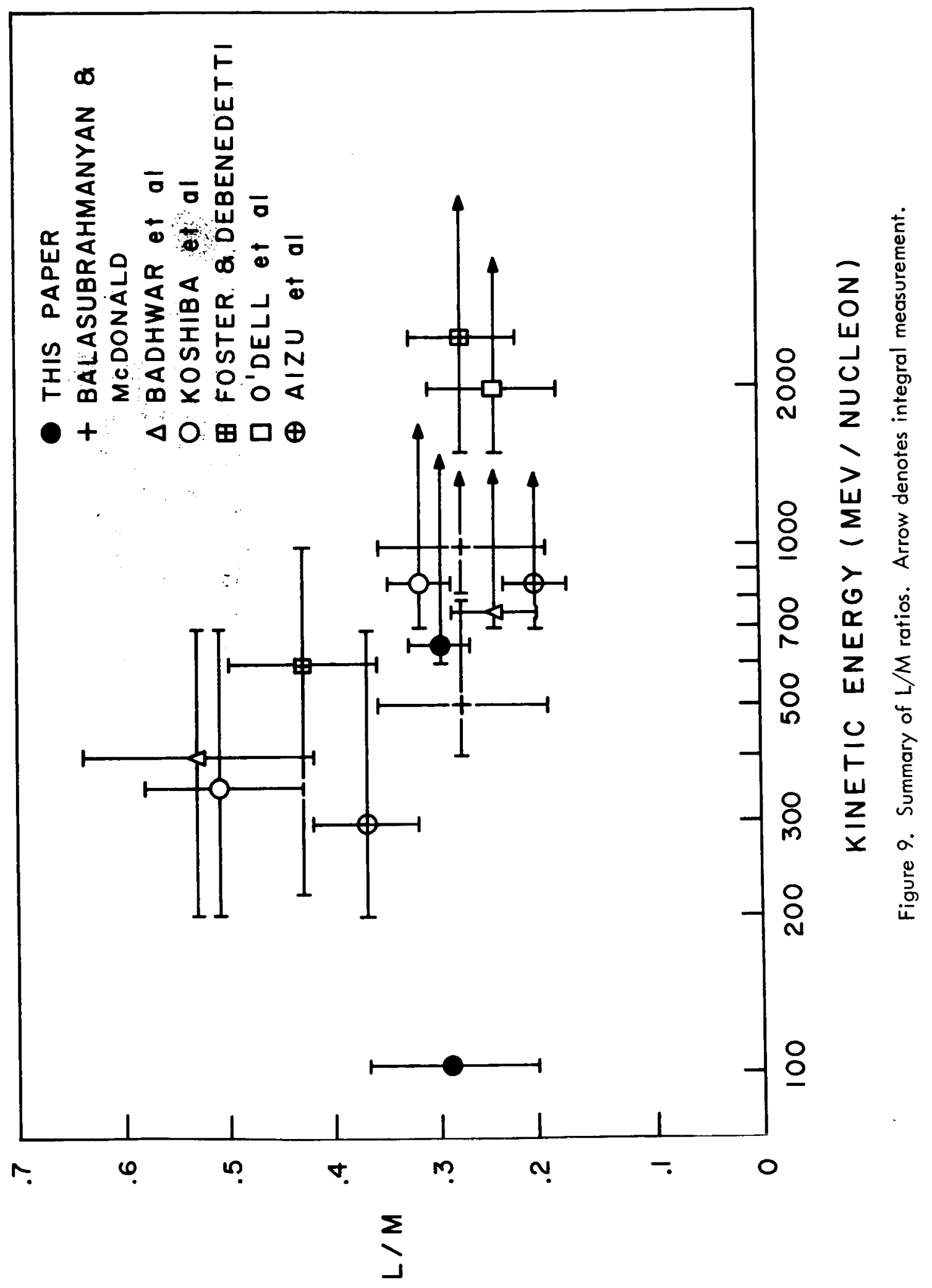

